\title{
Design and Development of Office Automation Management System based on ASP.net and SQL Server Technology
}

\author{
Xianghui Chen * \\ Qingyuan Polytechnic \\ Qingyuan, China \\ hdsdluyue@163.com \\ * Corresponding Author
}

\begin{abstract}
The office automation system should realize: the sharing of various information resources in the enterprise, strengthen the communication between the staff and improve the efficiency of the whole work. This paper first analyzes the development of office automation system tools and technology, include ASP.net and SQL Server. Then the paper discusses the research status of office automation system, and expounds the feasibility analysis, requirement analysis and system design of the system. Finally, the paper presents design and development of office automation management system based on ASP.net and SQL Server technology.
\end{abstract}

Keywords- Office Automation System; ASP.net; SQL Server; Visual Studio.NET; Management

\section{INTRODUCTION}

With the rapid development of the network, the network OA system is gradually concerned. Some large enterprise groups (such as Lenovo, Haier) are working to achieve high levels of network office automation, which will save a lot of human resources, save a lot of office expenses, greatly improve office efficiency.

The traditional office mode is mainly based on paper medium, in the wave of information revolution, it is clear that it has been far from meeting the needs of modern work and life with high efficiency and fast rhythm [1]. How to realize the information processing automation and office paper has gradually got the attention of people?

In the traditional office mode, because of the lack of important information transmission and work process, the information sharing and information integration among the various departments of the office can not be realized. In the daily office process, more prone to the following error: first, the information transfer and transaction of various departments are still done manually, two is the upper and lower levels and departments can not achieve collaborative work, it is difficult to communicate in time, three is the information and workflow, the lack of essential records, so that the work monitoring and assessment difficult.

With the development of information technology and the improvement of the application level of domestic information technology, in the process of office, the traditional work way and approval mode has been difficult to meet the new demand, the office automation is to improve the efficiency of government agencies and enterprises and institutions, the inevitable trend of standardization management. The benefits of the office automation system are: (1) the communication and exchange of information is smooth, the information communication is not smooth, and the communication platform is constructed, which can reduce the information's timeliness, shorten the distance between leaders and staff.

(3) Integrating information resources, realizing knowledge management, breaking the "information island", completely changing the file missing, managing and dispersing, searching for the phenomenon [2]. (4) Enhance cohesion, improve response capabilities, reduce management costs, promote management and progress, can train full information attention and participation awareness, inspire and use advanced information technology to enhance the enthusiasm, improve professional quality, and form a new corporate culture.

ASP is the abbreviation for Server Page Active. It is a web page that contains the code for the Script VB or Jscript script. When the browser to browse the ASP page, the Web server will generate the corresponding HTML code according to the request and then return to the browser, so that the browser is to see the dynamic generated web. ASP it can interact with the database and other procedures is a simple, convenient programming tool. After understanding the basic syntax of VBSCRIPT, it is clear that the use of the various components, properties, methods, you can easily write their own ASP system. ASP the format of the web file is ASP.

One of the major advantages of Server SQL is the tight integration of the mainstream client / server development tools and desktop applications. For example, you can access the Server SQL database in Basic Visual, C++ Access, Visual, Builder, Power, Delphi, and FoxPro Visual. In the development of database applications, you can use data access objects (DAO), remote data objects (RDO), ActiveX controls, DB OLE, ODBC, DB-Library and other development tools provided by the third parties to access the Server SQL database. Server SQL client applications can access the data from the server through the application interface provided by Server SQL. There are four main access methods: API OLE, DB Transact-SQL, ODBC, and DB-Library. For a client, these API can be used as a dynamic link library and communicate with the Server SQL server through the client's network library. 
Office automation is a new kind of office style of information society. It combines modern office and computer network. With the development of information technology, office automation plays an increasingly important role in the management and operation of the enterprise. Through the network, the organization's internal staff can work across time and place. Through the application of the exchange network in the office automation system, the information transfer is faster and more convenient, which greatly expands the management means, and realizes the operation of high efficiency. The paper presents design and development of office automation management system based on ASP.net and SQL Server technology.

\section{DEVELOPMENT TOOLS AND DATABASE DESIGN}

ASP.NET uses a hierarchical configuration system based on text, which simplifies the application of server environment and Web application. Because the configuration information is stored in the YISHION text form, it can be applied without the help of the local management tool [3]. This "zero local management" philosophy is also extended to the deployment of ASP.NET framework applications. Simply copy the necessary files to the server, and then deploy the ASP.NET framework to the server. Do not need to restart the server, even when the compiler code is deployed or replaced.

ASP is a technology developed by Microsoft, which requires the implementation of the Windows environment. The Web server used by PWS is Windows, while NT/2000 IIS is Windows95/98. Server SQL database system of the server running on the NT/2000 Windows system, responsible for creating and maintaining the table and index and other database objects, to ensure data integrity and security, can recover data in the presence of various errors. Client applications can run on the $9 x / \mathrm{NT} / 2000$ Windows system to complete all of the user interaction. After the data is retrieved from the server, you can generate a copy, so that you can keep it locally, you can also perform the operation.

Studio.NET Visual 2013 is to solve the most challenging software development needs and construction. .NET it is the second generation of Microsoft development tools for building and deploying powerful and secure connection.NET Microsoft software. Provides a powerful and perfect end to end tool is to design, development, debugging and deployment for the safety of the Microsoft Windows \& reg; and web applications. These applications are robust and easy to use.

ASP.NET provides tracking service that can be enabled in the application level and page level debugging. You can choose to view the information on the page, or use the application level of the tracking to view the information. In the development and application of the production status, ASP.NET supports the use of Framework.NET debugging tools for local and remote debugging. When the application is in production, the trace statements are left in the product code without affecting the performance. And Framework.NET integration: because.NET is part of the Framework ASP.NET, the entire platform functionality and flexibility of Web applications are available, as is shown by equation (1).

$$
W_{j}=\overline{\operatorname{span}\left\{\psi_{j, i}: i \in Z\right\}}, j \in Z
$$

Server SQL is widely used in many places, such as small businesses, large corporations, and the development of a program of love to use it to produce a desktop system for processing data. It is also often used to develop a simple WEB application that uses ASP technology to run in Information Services Internet. The more complex WEB applications use PHP/MySQL or SQL Server. ASP/Microsoft.

A wealth of programming interface tools, the use of Server SQL database application developers more flexible. Server Transact-SQL provides DB-Library, for C SQL, embedded SQL (ESQL) and other development tools, Transact-SQL and industry standard SQL language is compatible, and it is based on the expansion, so that it is more suitable for business processing needs. In addition, Server SQL 2008 also supports OLE, DB ADO, ODBC specification; you can use OLE, DB ODBC, ADO interface to access the Server SQL database.

Server SQL 2008 is a high performance client / server architecture for the relational database management system, supporting the maximum Web site and the enterprise online transaction processing (OLTP) and the performance of the data system [4]. Server SQL 2008 mainly has the following characteristics: (1) the real client / server architecture; (2) graphical user interface, make the system management more intuitive and simple, as is shown by equation (2); (3) a wealth of programming interface tools for users to design and provide a greater choice.

$$
p^{\prime}=W(p, D, \theta)=s\left(\begin{array}{cc}
\cos \theta & \sin \theta \\
-\sin \theta & \cos \theta
\end{array}\right) p+\left(\begin{array}{l}
d_{1} \\
d_{2}
\end{array}\right)
$$

Dynamic automatic management is optimization. Even if the Server SQL database administrator does not do any settings, Server SQL is also capable of automatically configuring the environment and user access in the running process to achieve the best performance, thereby reducing the administrator's work. Full Internet is technical support. Internet network development has become an important information release channel, Server SQL enhanced the Internet technology support, which in addition to retain the early version of the database Web publishing tool Web assistant, but also enhanced support for HTTP and XML technology, which makes the XML database system to access the Server SQL database system, and also extends the application of Server SQL in the field of data mining and analysis.

Community Starter Kit ASP.NET data access layer is used to connect Server SQL database, data access layer is implemented as a Server SQL storage process, Server SQL data sending and receiving are completed by the storage process, each database operations are stored, for data retrieval, insertion, update, or delete.

Server SQL is able to access Server/Jet SQL, Server SQL, Oracle, or any ODBC compliant database. Skilled software designers and data analysts to use it to develop application software, and some not skilled programmers and non programmers "advanced users can use it to 
develop a simple application software. Although it supports some object-oriented (OO) technology, it has not become a complete object-oriented development tool.

Server SQL 2008 and Windows Microsoft 2000 organic integration, so you can use the Windows 2000 (Directory Active) function to focus on Server SQL management, greatly simplifying the system management in large enterprises [5]. In addition, the integration with Windows 2000 also enables Server SQL to fully utilize the services provided by the operating system (such as security management, event logs, performance monitors, memory management and asynchronous $\mathrm{I} / \mathrm{O}$, etc.), thus enhancing the functionality of the Server SQL database system, and only need to take up very little system resources.

$$
g(n)=(-1)^{n-1} \tilde{h}(-n+1)
$$

Server SQL 2008 is a network database management system, is the most powerful relational database system, its production tasks is to store, manage and provide data to meet the needs of client connection and storage data, powerful, convenient, can establish data types, as is shown by equation(3), data warehouse and data tables, database query, delete, update, add, storage, index and database, Transact-SQL programming, storage process, trigger, user defined functions and transactions, Server SQL 2000 management, database development and application.

Compatibility with existing ASP applications: ASP and ASP.NET can run in parallel on the Web IIS server and are not mutually compatible; it will not happen because of the possibility of installing ASP.NET and leading to the collapse of existing ASP applications. ASP.NET only deals with files with .Aspx file extension. File extensions with .Asp files continue to be processed by the ASP engine. However, it should be noted that the session state and application state are not shared between ASP and ASP.NET pages

\section{OFFICE AUTOMATION MANAGEMENT SYSTEM AND REQUIREMENT ANALYSIS}

Most of the small and medium enterprises have been established so far, the office mode is the traditional office mode and mainly based on paper media, in the wave of the information revolution, it is clear that the modern work and life of the modern work and life can not meet the needs of high efficiency and fast rhythm [6]. How to realize the information processing automation and office paper is gradually got the attention of the company leaders?

The existing network office automation system and large information management system, the enterprise business process reengineering or file transfer function are core functions. At the same time, the office of the enterprise is the process of a file transfer; all the office affairs can be abstracted into a database object.

The development of network office system's market prospects is broad. Large enterprises need to high level of network office automation, they tend to choose large software companies to cooperate in the development, the development costs and maintenance costs are also very high. These high costs are not the majority of small and medium enterprises can afford. Small and medium enterprises have a large demand for low cost OA systems, and we can develop these low cost OA systems to meet this demand, as is shown by equation (4) [7].

$$
\mu_{h}^{(0)} \leftarrow \frac{1}{\left|S_{h}\right|} \sum_{x \in S_{h}} x, h=1, \cdots, k ; t \leftarrow 0 ;
$$

The company is widely used to improve the work efficiency of the staff, but in the modern work environment that needs a lot of employees work together, the company needs to improve the overall work efficiency. The use of network communication infrastructure and advanced network application platform, building a safe, reliable, open and efficient information network and office automation, information management system, to provide management with modern office conditions and rich comprehensive information services, real file management automation and office automation to improve office efficiency and management level, to achieve the company's daily business work standardization, electronic, standardization, information online inquiries, borrow. Final implementation of "no paper" is office.

Through the website of the enterprise internal information for a comprehensive automated management, greatly improving the enterprise's office efficiency. Through the system of enterprise production and management of the data in the process of comprehensive management and statistics, to avoid the various problems arising from the handling of various types of data, improve the economic benefits of enterprises, to provide a large number of business decisions, the authority of the data, so that the management of enterprises to scientific, systematic and scientific.

It should be said that no one has the advantage of the emergence of the computer to the office staff, but for modern office automation, its purpose has been not limited to reduce the workload of staff, but the staff from the tedious work of the work, and more energy to focus on the most important things, so as to enhance the efficiency of enterprises, enhance the comprehensive competitiveness of enterprises

In this kind of office mode, enterprise's organization structure can be simplified, each department in the information sharing based on collaboration, easy to clear the various departments and even the responsibility of each employee, and the decision-making layer can quickly integrate from all aspects of information, and based on the development of strategic decision-making. In this sense, in the increasingly fierce market competition today, office automation has become a tool, a tool, and become the lifeblood of the survival and development of enterprises.

OA system to achieve: $A$, the enterprise information resources sharing; $\mathrm{B}$, strengthen communication between employees, improve the overall work efficiency; C, to lead a variety of useful data, to facilitate the leadership of the company's situation in a timely manner to provide decision support.

\section{DESIGN AND DEVELOPMENT OF OFFICE} AUTOMATION MANAGEMENT SYSTEM BASED ON ASP.NET AND SQL SERVER

The development of office automation system based on the small and medium sized enterprises has adopted the 
advanced technology and the development method which is in line with the practical application [8]. ASP.net architecture is a What (R) Studio.net Microsoft Visual development environment for development, WYSIWYG (You See Is What You Get, see the. These are only a small part of the ASP.net's powerful software support. ASP.net makes it very simple to run some of the very common tasks such as the form of authentication, distribution system, and web configuration of the client's identity verification.

ASP.NET uses a text based, hierarchical configuration system that simplifies the work that will be applied to the server environment and Web applications. Because configuration information is stored as plain text, it can be applied without the help of local management tools. Any changes in the configuration file can be automatically detected and applied to the application [9]. For more information, see the ASP.NET configuration.

Into the available programming language, it is in Office Microsoft furniture, Basic Visual. Two databases into the message database COM component are provided: the legacy data access object (DAO), with access to and can only provide the new ActiveX data objects (ADO). Server SQL is easy to be applied to a small project, but the size of the project is invalid [10]. All database query, form and report is stored in the database, and the ideal coordination with the relevant model, there is no possibility of a physical organization of the class, as is shown by equation (5).

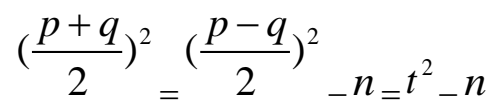

The main process of the system is the system administrator or ordinary users to enter the system through the login module, then you can choose the corresponding function to deal with their own information. The main functions of the system are summarized as follows:

(1) User identity verification module, judging the current logged in user is the type, if it is ordinary users and then enter the front office interface, and the corresponding restrictions. If it is the system administrator to enter the background management interface.

(2) User identity management module, the module is mainly for the user identity to add, modify, delete. The function of the system is the main system administrator.

(3) The communication record management module, the function of the module is the employee address book management (add, modify, delete). The function of the system is the main system administrator.

The system is based on the three tier architecture. In general, the three-layer structure is divided into Presentation (layer Application), layer, data access layer (Data), data access layer (access layer). The purpose of distinguishing the level is to "high cohesion and low coupling". Layered structure is the most common and the most important one in software architecture design. Microsoft recommended hierarchical structure is generally divided into three layers, from the bottom to the top are: data access layer, business logic layer (also known as the field layer), said layer.
In the process of information structure and conceptual model, the user needs to be abstracted from the requirement analysis. In order to describe the concept model of the database structure, the E-R diagram is used to describe the entity relationship of the database. In employee information management module, a company can have more than one company employees, but a company employee can only have a company position. As a result, there is a relationship between the company's position and the system user, a company can have a number of employees, but company employees can only belong to a company, so the company is a multi relationship between the company and the system.

The system is based on the three layer structure, according to the actual development process to develop. Including the following: solid layer function development (Model): the use of Linq to achieve the mapping of the database table, and the operation of data; DAL data access layer function development: to add, modify, three layers, and filter data. LL business logic layer function development: to achieve the logical judgment of the data, verify and other functions. UI representation layer function development (UI): the realization of data presentation, data entry functions, and operation of the process. The purpose of the three layers is to distinguish the level of the purpose of "high cohesion and low coupling".

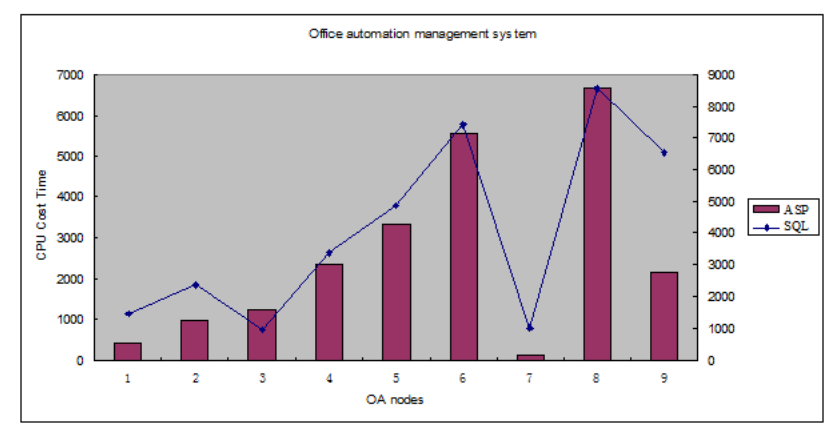

Figure 1. Office automation management system based on ASP.net and SQL Server.

A combination of structure and prototype method based on the office automation system of small and medium sized enterprises. The advantage of this method is that it takes into account the characteristics of the development of the structure of the development process control and the characteristics of the short development cycle, quick response. Using the combination of the two forms of the development of the system, the use of structured life cycle method of design ideas, namely, topdown, from the overall to the part, a reasonable division of the structure of the system, the design of the database model.

In the system analysis and preliminary design, we use the prototype method to make the original model, according to the actual situation, continue to carry on the detailed design and implementation and transformation, system maintenance and evaluation phase of the system. Rational use of comprehensive method is the development process more flexible, and it is more often to achieve better results. 


\section{SUMMARY}

The paper presents design and development of office automation management system based on ASP.net and SQL Server technology. Based on the office automation system of small and medium enterprises, the whole system is divided into several functional modules, and according to the actual ability of the development team members to allocate the corresponding module, this system functions parallel to the method of system development and management.

\section{REFERENCES}

[1] Lingbo Kong, Kaichao Yu, Jianhua Zhang, Yanfang Liu, "Research on PVP Exam System Based on ASP", IJACT, Vol. 5, No. 1 , pp. $757 \sim 766,2013$

[2] Qing-jie Liu, Xiao-ying Wang, Wei Zhang, "Design and Implementation of MSQL Server Database System Model", AISS, Vol. 4, No. 12, pp. $124 \sim$ 131, 2012.
[3] Wei Min, "A Research on Statistical Information Applied to Tourist Traffic and Transport System Design Based on ASP. NET", JCIT, Vol. 6, No. 1, pp. $147 \sim$ 156, 2011.

[4] Zhi-ping Zhou, Jiang- wei Wu, "The Development of Data Warehouse System Based on Msql Server", JDCTA, Vol. 6, No. 12 , pp. $124 \sim 131,2012$.

[5] Hamid Turab Mirza, Ling Chen, Gencai Chen , "Practicability of Dataspace Systems", JDCTA, Vol. 4, No. 3, pp. $233 \sim 243,2010$.

[6] Preecha Noiumkar, "The SQL Injection Attacking Prevention Applying the Design Techniques of IPTABLES", JCIT, Vol. 7, No. 22, pp. $66 \sim 75,2012$.

[7] Gu Wei, Hu Rui, "Design and Implementation of Competitiveness Evaluation System for Metallurgical Enterprises Based on MySQL and JSP", JCIT, Vol. 8, No. 3, pp. 610 618, 2013.

[8] Atefeh Tajpour, Suhaimi Ibrahim, Maslin Masrom, "SQL Injection Detection and Prevention Techniques", IJACT, Vol. 3, No. 7, pp. $82 \sim 91,2011$.

[9] Somboon Anekritmongkol, Kulthon Kasemsan, "Boolean Normalized By SQL for Association Rule Mining", IJACT, Vol. 5, No. 14, pp. $138 \sim 149,2013$.

[10] Eun Hong Cheon, Zhongyue Huang, Yon Sik Lee, "Preventing SQL Injection Attack Based on Machine Learning", IJACT, Vol. 5 , No. 9, pp. $967 \sim 974,2013$. 